\title{
Investigating of Turkey Champion Clubs' Financial Performance Using Bootstrap and Jackknife Methods
}

\author{
Tolga Zaman, Kamil Alakus, Hasan Bulut \\ Department of Statistics, Faculty of Science and Arts, Ondokuz Mayıs University, Samsun, Turkey
}

Email address:

tolga.zaman@omu.edu.tr (T. Zaman)

\section{To cite this article:}

Tolga Zaman, Kamil Alakus, Hasan Bulut. Investigating of Turkey Champion Clubs' Financial Performance Using Bootstrap and Jackknife Methods. American Journal of Theoretical and Applied Statistics. Vol. 4, No. 2, 2015, pp. 58-63. doi: 10.11648/j.ajtas.20150402.13

\begin{abstract}
This study attempts to investigate using bootstrap and jackknife methods how accurate Turkey's teams, being champion since super league was established, use their financial expenditure for the scores when they were consecutively in league between 2006-2007 and 2013-2014 seasons. In this context, the champion clubs' financial performance values said eight seasons were obtained by dividing clubs' total expenditure in each season to scored total points in current season. Also, the results obtained by bootstrap and jackknife methods were compared on the basis of error mean square. Thus, it was judged that bootstrap method gives better results than jackknife method. All statistics of bootstrap and jackknife methods were calculated by the aid of the R packaged software. Turkey's clubs which had championship: Besiktas, Bursaspor, Fenerbahçe, Galatasaray and Trabzonspor.
\end{abstract}

Keywords: Resampling, Bootstrap, Bootstrap Confidence Interval, Jackknife, Bias, Error Mean Square

\section{Introduction}

In statistics, the uses of some methods are seen as demanding and frightening by most researchers. This attitude of researchers increases possibility of making a mistake. With the dissemination of statistical software packages, it can be taken more accurate decisions about the validity and conformity of the analysis. In literature, bootstrap and jackknife methods being resampling methods are used quite common in applied statistic. The basic idea in bootstrapping is that the actual data are resampled to produce a large number of data sets [1]. Thus, it can be obtained information as much as possible from samples in small data sets. Bootstraps is a method that is away from excessive intensive mathematical formulas, with limited hypothesis, quite easy to understand and implement [2]. This technique gives reliable results, especially in case of remaining incapable of the hypotheses of the statistical methods. Bootstrap method, as in probability [3], is used in many fields such as confidence intervals, hypothesis testing, regression and time-series analysis [4]. The basic behind jackknife lies in systematically recomputing the statistic estimate based on subsets of observations, which are obtained from the original set of the observations but leaving one observation out at a time. Pseduevalues are obtained by leaving out each observation in data set. Pseduevalues observed can be considered as if they had independent and same range [5].

However, even if researchers are aware of resampling methods, the limited software packages hinder implementation of them. Even with awareness of these concepts and access to software, some researchers hesitate to apply these methods seen as new and detail. Because, while newer techniques are face with criticisms and seem to be far from theoretical basis, traditional methods are perceived as have theoretical support and empirical substantion [6].

In terms of global economy, football has significant economic consequences. These results also take effects on economies at various levels. Sports mentioned, football primarily comes to mind the world over. Because 3.5 billion people are interested in football. After the 1990s, there have partly been changes in the structural characteristics of football. Football is no longer just a sport, has become an industrial business line [7]. The transformation of football clubs from non-profit into profit-oriented organizations had radical changes for the club's position in national and international arena. Some concepts such as financial performance and budgeting -not so frequently mentioned have come into prominence [8]. After the 1990s, the football clubs have to redefine their positions in marketplace and adapt their decision-making-process to new regulations [9]. 


\section{Methods}

\subsection{Bootstrap Method}

Bootstrap method was first proposed by Bradley Efron in 1979 as an alternative to jackknife which is another resampling method. In 1993, it was developed by Efron and Tibshirani [10-4]. The bootstrap is a simple and straightforward method for standard deviation, confidence interval and nonparametric estimation problem. The basic logic of the method lies producing new data sets by resampling with replacing observations in way aleatoric from any size of available data set. Thus, it can be obtained information as much as possible from available data set. Firstly, a bootstrap sample is composed by selecting samples with replacement from original sample. Then, this process is repeated a large number of times and so, it is calculated confidence interval of estimator and statistical significance of test [11-12]. Also, the parameters can be estimated independent from hypothesis of normal distribution in this method. Even incomplete and small data sets can be used to make statistical inference [3]. Schematic diagram of the method is as follows [13].

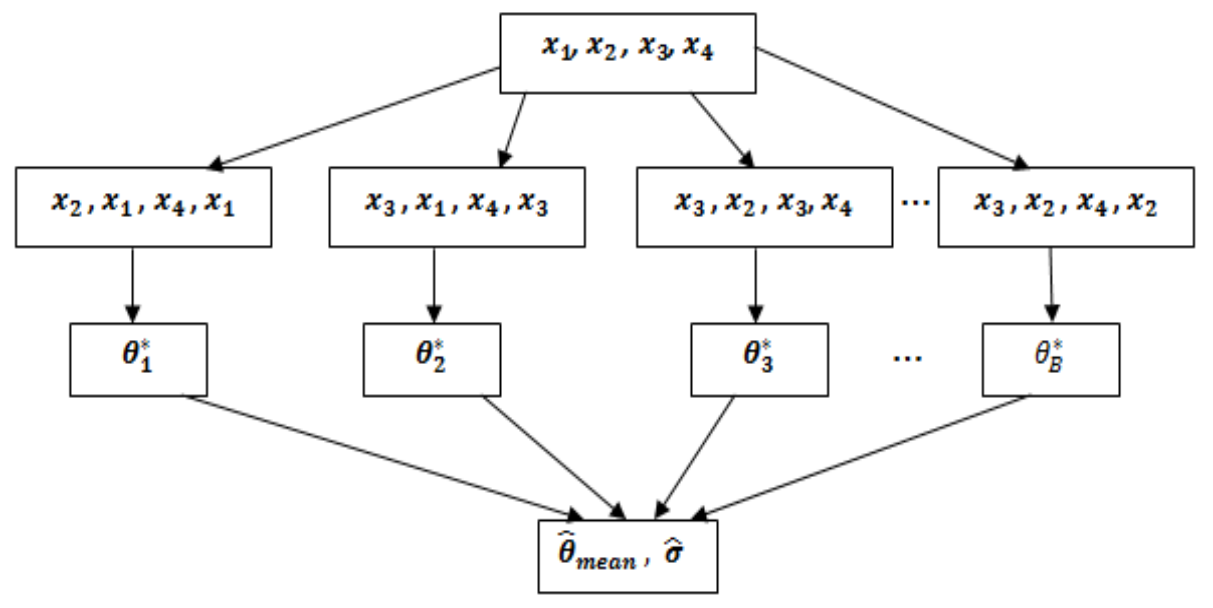

Figure 1. Schematic diagram of Bootstrap method.

It is possible to generate approximately confidence intervals with bootstrap method [10]. Generally, it gives more information than point estimate of any $\theta$ parameter [13].

Bootstrap Standard Intervals: This confidence interval bases parametric model of confidence interval and may be written as follows.

$$
\widehat{\theta}-z_{1-\frac{\alpha}{2}} \times S E_{\widehat{\theta}}, \hat{\theta}+z_{\frac{\alpha}{2}} \times S E_{\widehat{\theta}}
$$

In here, $S E_{\widehat{\theta}}$ is standard error of $\theta$ statistic obtained by bootstrapping.

Bootstrap Percentile Interval: This interval is valid when the bootstrap distribution is about normal. If sampling distribution of bootstrap statistic is almost normal, standard normal and percentile interval will give close results to each other. For example, for $n \rightarrow \infty$, in the central limit theorem histogram of bootstrap is normal and thus, both confidence intervals give close results. However, it is stated that it will go away from normal in small samples. And with this, standard normal intervals and percentile confidence intervals may be different.

$B C \alpha$ (Bias-Corrected and Accelerated): $\mathrm{BC} \alpha$ confidence interval difficult to calculate in theoretically has two important theoretical advantages. The first one, it is suitable for function transformation, the other one is related to the accuracy of $\mathrm{BC} \alpha$ confidence intervals. The $\mathrm{BC} \alpha$ confidence interval does not require any hypothesis. For these reasons, it is a method frequently used in researches. Yet, it cannot be said that this method is the best one and impossible to do the better.

\subsection{Jackknife Method}

Jackknife method was first used to remove statistically biases by Ouenouille in 1956. Tukey (1958) [14] suggested a simple approach, jackknife estimates based on removing data and then recalculating the estimator provides a general purpose statistical tool that is both easy to implement and solves a number of problems.

The motivation behind the jackknife is as follows. If $\bar{x}$ denotes the mean for a sample of size $n$, we can also compute the sample mean when the jth data point is removed (or jackknifed),

$$
\bar{x}_{-j}=\frac{1}{n-1} \sum_{i \neq j}^{n} x_{i}
$$

Observe that if we know both $\bar{x}$ and $\bar{x}_{-j}$ we can compute the value of the jth data point as

$$
x_{j}=n \bar{x}-(n-1) \bar{x}_{-j}
$$

Suppose we wish to estimate some parameter $\theta$ as a statistic of the $n$ data points,

$$
\hat{\theta}=\emptyset\left(x_{1}, x_{2}, \ldots, x_{i-1}, x_{i}, x_{i+1}, \cdots, x_{n}\right)
$$

Motivated by Equation (1), let the jth partial estimate of $\theta$ be given by the the estimate computed with data point $x_{j}$ removed, 


$$
\hat{\theta}_{j}=\emptyset\left(x_{1}, x_{2}, \ldots, x_{i-1}, x_{i+1}, \cdots, x_{n}\right)
$$

By analogy with equaiton (2), define the jth pseudovalue by

$$
\hat{\theta}_{j}^{*}=n \hat{\theta}-(n-1) \hat{\theta}_{j}
$$

These pseudovalues assume that same role as the $x_{j}$ in estimating the mean, hence the jackknife estimate of $\theta$ is given by the average of the pseudovalues,

$$
\hat{\theta}^{*}=\frac{1}{n} \sum_{i=1}^{n} \hat{\theta}_{i}^{*}
$$

An approximate sampling error for $\hat{\theta}^{*}$ can be obtained from the sample variance of the pseudovalues,

$$
\operatorname{Var}\left(\hat{\theta}^{*}\right)=\frac{\operatorname{Var}\left(\widehat{\theta}_{j}^{*}\right)}{n}=\frac{\sum_{j=1}^{n}\left(\widehat{\theta}_{j}^{*}-\widehat{\theta}^{*}\right)^{2}}{n(n-1)}[15]
$$

\subsection{Real Data Application}

In this section, it is aimed to investigate how accurate Turkey's teams, being champion since super league was established, use their financial expenditure for the scores when they were consecutively in league between 2006-2007 and 2013-2014 seasons. Because now, UEFA can exclude from European competitions with regulations of UEFA financial fair play, if the clubs do not use their budgets in balanced manner. In consideration of these regulations, we wanted to analyse how much the champion football teams in our country spend and in addition to this, how accurate they spend. In research, we were interested in how they spend their budget in last 8 seasons. We determined variable as total expenditure used to score a point by dividing clubs' total values in last 8 seasons to total score in current season. It is hoped to show that the most successful club scores the points with the least expenditure. Next, according to variable, it was obtained statistics of bootstrap and jackknife and gotten some results about the use of finances for their clubs in the most correct way. The interpretations were made with the help of these results.

Statistic we will use in here is mean. The bootstrap and jackknife values of statistics were obtained and made interpretations.

Table 3.1. Financial Performance Index

\begin{tabular}{llllll}
\hline Seasons & Bjk & Bs & Fb & Gs & Ts \\
\hline $06-07$ & 0.8981 & 0.5311 & 1.1636 & 1.0643 & 1.2002 \\
$07-08$ & 0.7236 & 0.8184 & 1.1692 & 0.9310 & 0.8673 \\
$08-09$ & 1.0035 & 0.5086 & 1.4918 & 1.4943 & 0.7815 \\
$09-10$ & 1.3734 & 0.7013 & 1.4095 & 2.0273 & 1.0614 \\
$10-11$ & 1.9861 & 1.0582 & 1.6888 & 2.1717 & 0.9585 \\
$11-12$ & 2.1882 & 1.0520 & 1.9125 & 1.5571 & 1.5589 \\
$12-13$ & 1.7888 & 0.9755 & 2.4795 & 2.0915 & 1.8641 \\
$13-14$ & 1.6218 & 1.025 & 1.7878 & 2.1715 & 1.3113 \\
\hline
\end{tabular}

Table 3.1 demonstrates variable which was obtained by dividing Turkey's champion football teams' total values between 2006 and 2014 seasons to total score gotten in mentioned season. With the use of this variable, the clubs' statistics of bootstrap and jackknife are obtained. First, we let get the Besiktas' bootstrap and jackknife statistics by using mean statistic. The calculations were carried out with the help of R packaged software [16-17]. For example, for Bjk it was obtained from original data set 10000 bootstrap repeats.

ORDINARY NONPARAMETRIC BOOTSTRAP

Call:

$\operatorname{boot}($ data $=$ bjk1, statistic $=$ function $(a, i)$ mean $(\operatorname{abjk}[i])$, $\mathrm{R}=10000)$

Bootstrap Statistics: original Bias $\quad$ Std. Error $\quad$ MSE

$\mathrm{t} 1 * 1.447938 \quad 0.002537901 \quad 0.17644290 .0311385379$

If we are to explain what the meaning of these results are; Original $=1.447938$ is mean of eight observations belonging to original data set.

As for Bias $=0.002537901$ value, it is expressed as bootstrap statistic of 10000 values concerning with mean.

Standard Error $=0.1764429$ value is standard error of 10000 bootstrap statistic belonging to mean.

$M S E=$ Var + Bias $^{2}=0.1764429^{2}+0.002537901^{2}=$ 0.0311385379 was calculated as.

For example; it can also be obtained bootstrap mean of mean. As follows;

$\operatorname{Bias}($ mean $)=$ mean $($ boot $)$-original $($ mean $)$ $0.002537901=$ mean(boot) -1.447938

mean $($ boot $)=1.450475901$ value was obtained as 10000 bootstrap mean of mean.

The bootstrap statistics of Besiktas obtained by R packages software were gotten for other 4 clubs in the same way and made interpretations.

All mentioned 5 clubs' bootstrap statistics are shown in Table 3.2.

Table 3.2. Bootstrap Statistics of Financial Performance Index

\begin{tabular}{lllll}
\hline Clubs & Original & Bias & $\begin{array}{l}\text { Std. } \\
\text { Error }\end{array}$ & $\begin{array}{l}\text { Mean Square Error } \\
\text { (MSE) }\end{array}$ \\
\hline $\mathrm{Bjk}$ & 1.447938 & 0.002537901 & 0.1764429 & 0.0311385379 \\
$\mathrm{Bs}$ & 0.833762 & 0.000716216 & 0.0758154 & 0.0057484908 \\
$\mathrm{Fb}$ & 1.632837 & 0.001111501 & 0.1448863 & 0.0209932753 \\
$\mathrm{Gs}$ & 1.688512 & 0.003096294 & 0.1650633 & 0.0272554800 \\
$\mathrm{Ts}$ & 1.200400 & 0.000676971 & 0.1224900 & 0.0150042583 \\
\hline
\end{tabular}

Upon analysed Table 3.2, the most financially homogeneous team in last 8 seasons is Bursaspor which has the smallest mean square error. Bursaspor is respectively followed by Trabzonspor, Fenerbahce, Galatasaray and Besiktas. It is concluded that the clubs known as the three greatest of the Turkish football spend more money to score a point. These greatest clubs, Besiktas, Fenerbahce and Galatasaray, spend more generously their high incomes than their competitors and thus, it is seen that they take place in top positions compared to Anatolian clubs. Even if they had not had high income, unlike other Anatolian clubs, Bursaspor and Trabzonspor were managed to finish league as a 
champion by spending their budgets much more carefully and consciously than the three greatest clubs. Although the high incomes facilitate the achievement, the research carried out that clubs satiably accomplished with homogeneity and stability in financial expenditure, a modest budget in last 8 seasons.

Furthermore, if we analyse the plot graphics of mentioned 5 clubs;

For respectively Bjk, Bs, Fb, Gs, Ts; Normal plot graphics and the distribution of bootstrap repeats of financial performance index are as follows:

\section{Histogram of $\mathbf{t}$}

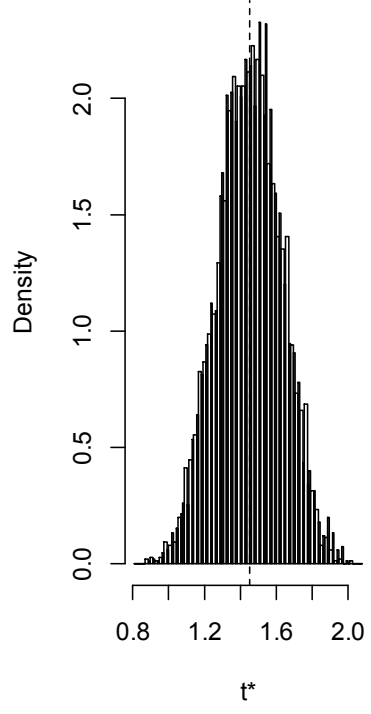

(a)

Histogram of $t$

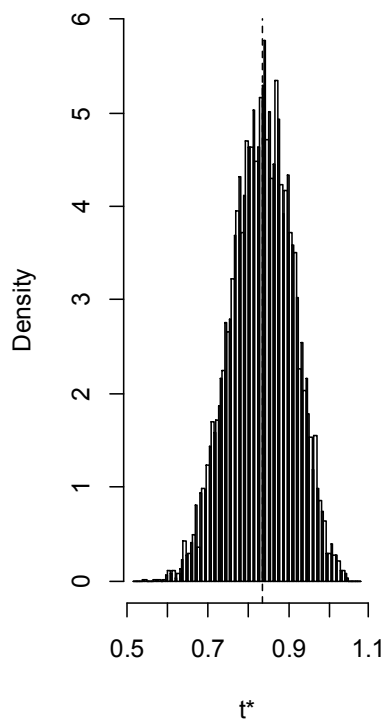

(b)

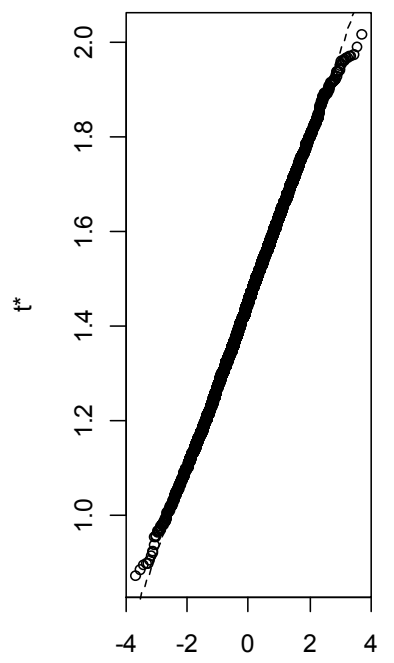

Quantiles of Standard Normal

$\left(a^{\prime}\right)$

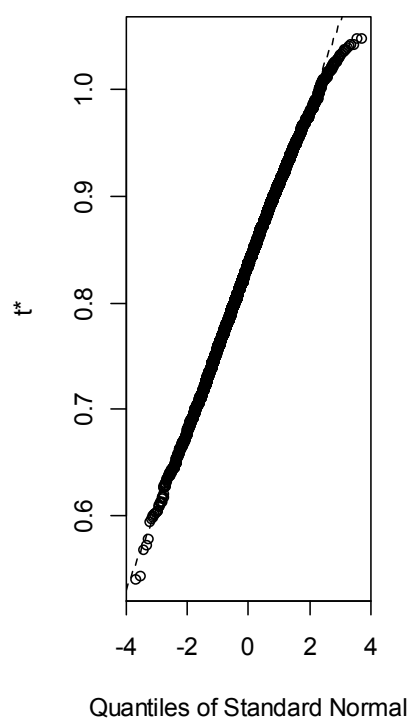

$\left(b^{\prime}\right)$

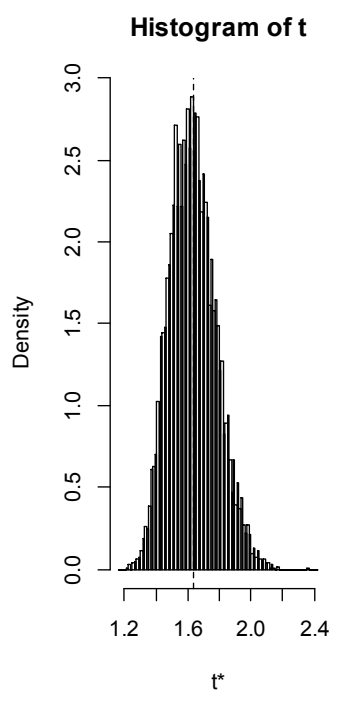

(c)

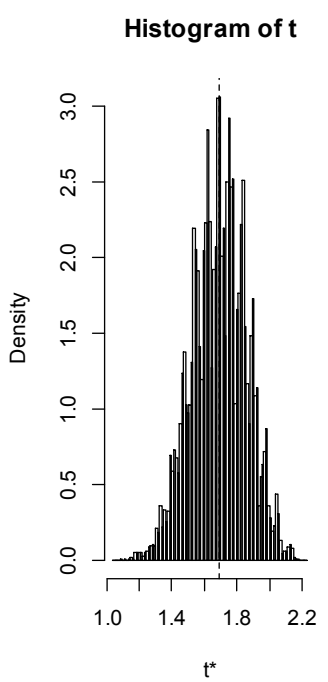

(d)

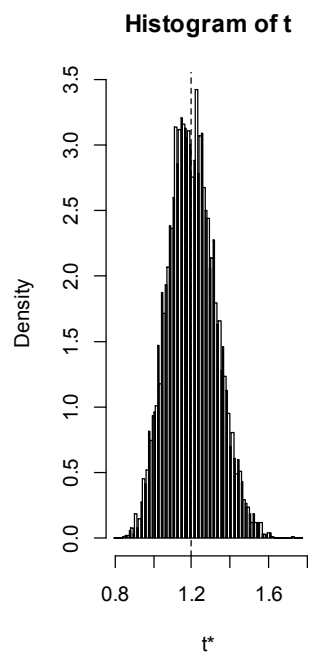

(e)

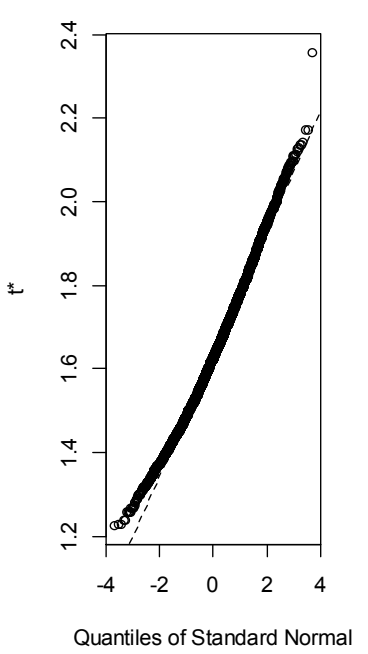

$\left(c^{\prime}\right)$

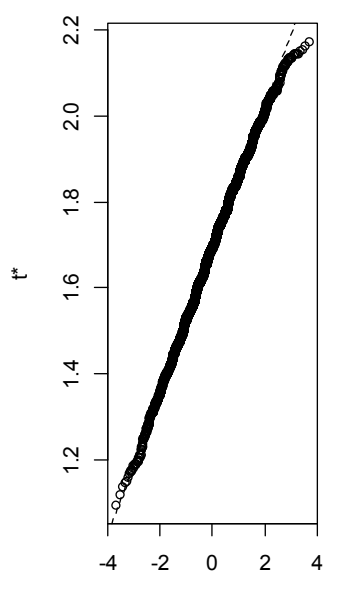

Quantiles of Standard Norma

$\left(d^{\prime}\right)$

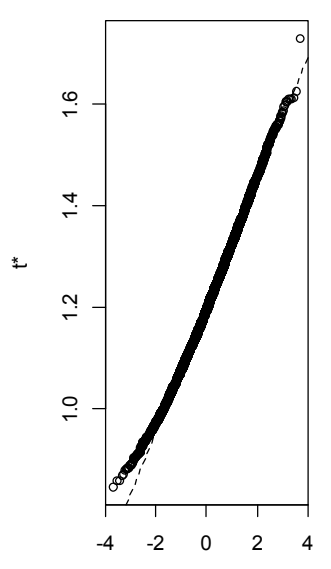

(e')
Graph 1. Distrubution of Bootstrap Repeat of (a) Bjk, (b) Bs, (c) Fb, (d) Gs, (e) Ts Club's Financial Performance Index and Bootstrap Normal $Q$ Chart of (a) Bjk, (b)Bs, (c) Fb, (d) Gs, (e)Ts Club's Financial Performance Index 
In these graphics, the left one shows the normality of data and the right one also shows how far observation values deviate from normality. Namely, the plot graphics indicate how far the estimated values of 10000 bootstrap repeats deviate from normal distribution. It is hoped that estimations related to all calculated bootstrap repeats are to as straight line on graph line. When plot graphics of five clubs are analysed together, almost all clubs can be evaluated as normal. Now, let's evaluate bootstrap confidence intervals by presenting a table.

Table 3.3. Bootstrap Confidence Intervals of Financial Performance Index

\begin{tabular}{lllll}
\hline Clubs & Normal & Basic & Percentile & BC $\boldsymbol{\alpha}$ \\
\hline $\mathrm{Bjk}$ & $1.11-1.79$ & $1.09-1.79$ & $1.10-1.79$ & $1.10-1.79$ \\
$\mathrm{Bs}$ & $0.68-0.98$ & $0.69-0.98$ & $0.68-0.98$ & $0.67-0.97$ \\
$\mathrm{Fb}$ & $1.35-1.92$ & $1.32-1.89$ & $1.38-1.94$ & $1.40-1.98$ \\
$\mathrm{Gs}$ & $1.36-2.01$ & $1.38-2.01$ & $1.37-2.00$ & $1.33-1.98$ \\
$\mathrm{Ts}$ & $0.96-1.44$ & $0.95-1.42$ & $0.98-1.45$ & $0.99-1.48$ \\
\hline
\end{tabular}

Firstly, we can analyse normal and percentile confidence intervals. If confidence interval of bootstrap repeats is to normal, these two intervals give close values to each other. Upon analysed actually plot graphics of mentioned 5 clubs, it was obtained almost normal graphics. Normal and percentile confidence intervals gave supporting information in here. Bursaspor is seen as a team which has the closest confidence intervals to each other. However in literature, there is information that $\mathrm{BC} \alpha$ confidence interval gives the best results, we will interpret in consideration of $\mathrm{BC} \alpha$ confidence interval.

In this study, it is considered that financial expenditure of teams involving $\mathrm{BC} \alpha$ confidence interval is optimal for financial performance index belonging to mentioned five clubs. For instance, this index is 0.70 in 2009-2010 seasons when Bursaspor became champion. This value is within obtained confidence limits (0.67-0.97). Therefore, Bursaspor's super league championship is an indication of success obtained by Bursaspor administration with financial expenditure in 2009-2010 seasons. In this context, it is concluded that teams spent generously their budgets when index fell below confidence interval or above it and thus, clubs were forced to themselves financially.

Let's get the results of jackknife technique; it is left one observation out of the number of $n$ observations and then sampled a new one with $n-1$ unit in jackknife method. In this way, only the number of $n$ different samples can be obtained. If we want to summarize the results of jackknife technique with a table, it is as follows;

Table 3.4. Jackknife Statistics of Financial Performance Index

\begin{tabular}{lllll}
\hline Clubs & Original & Bias & Std. Error & $\begin{array}{l}\text { Mean Square Error } \\
\text { (MSE) }\end{array}$ \\
\hline Bjk & 1.447938 & 0 & 0.189646724 & 0.03596588 \\
$\mathrm{Bs}$ & 0.8337625 & 0 & 0.081260014 & 0.00660319 \\
$\mathrm{Fb}$ & 1.632837 & 0 & 0.153775778 & 0.02364699 \\
$\mathrm{Gs}$ & 1.688512 & 0 & 0.177408680 & 0.03147384 \\
$\mathrm{Ts}$ & 1.200400 & 0 & 0.12994421 & 0.01688550 \\
\hline
\end{tabular}

The basic logic of Jackknife technique is to calculate sample statistics with other observations by leaving one observation value out at a time. Shortly, the same test is repeated by leaving one out at every stage. Thus, jackknife method also has an effect on reducing bias. When the results of bootstrap and jackknife statistics were compared, jackknife method reduced bias even eliminated. As it is known, variance is equal to mean square error in unbiased estimates.

Upon analysed table 3.4, in terms of scored points, the most homogenous team order is the same as order obtained by bootstrap method in last eight seasons.

Table 3.5. MSE of Bootstrap and Jackknife Statistics

\begin{tabular}{lrr}
\hline Clubs & $\boldsymbol{M S E}_{\text {Воот }}$ & $\boldsymbol{M S E}_{\text {JАСКк }}$ \\
\hline $\mathrm{Bjk}$ & 0.0311385379 & 0.03596588 \\
$\mathrm{Bs}$ & 0.0057484908 & 0.00660319 \\
$\mathrm{Fb}$ & 0.0209932753 & 0.02364699 \\
$\mathrm{Gs}$ & 0.0272554800 & 0.03147384 \\
$\mathrm{Ts}$ & 0.0150042583 & 0.01688550 \\
\hline
\end{tabular}

Upon compared these methods in consideration of table 3.5, mean square error of bootstrapping is smaller than MSE of jackknife method. Thus, it is determined that bootstrap method is more effective than the other one. However, for both methods, the order of using clubs' financial budgets for their benefits has not changed.

\section{Conclusion and Suggestions}

By means of a great number of bootstrap repeats, the analyses show to achieve the overall results. The biggest advantage of resampling methods, particularly bootstrap method, is to treat with small original samples for much more observations. Actually, the calculation of standard error and mean square error of 10000 trials is quite difficult. But with developed computer technology, this difficulty eliminates. The calculations were made by means of $R$ packaged software. A lots of analyses related to bootstrap method can be made with the help of this software.

In application, it was obtained mean square error of teams by the help of index and so, clubs' financial structures were analysed. Furthermore, bootstrap confidence intervals and bootstrap plot graphics were carried out and interpreted from different perspectives. Next, it was obtained results of jackknife technique by being based on mean statistic and with the same index. Also, it was observed that jackknife technique eliminated bias. Beside this, it was inevitable some growth in standard error.

This such study related to champion football teams in super league and done by using two resampling methods, bootstrapping and jackknifing, is not before seen in literature. Thus, the results and interpretations of this study may be beneficial to later studies. 


\section{References}

[1] Sacchi, M. D., 1998. A bootstrap producure for highresolution velocity analysis. Geophysics, Vol: 63(5).

[2] Simon, J. L., and Bruce, P., 1991. Resampling: A tool for everyday statistical work. Change, 4(1), 22-32.

[3] Takma, Ç., and Atıl, H., 2003. A Study on Bootstrap Method and Its Practice 1. Probability and Bootstrap Method. Journal of Ege University Faculty of Agriculture., 40(3): 89-96

[4] Efron, B. and Tibshiranni, R., 1993. An introduction to the Bootstrap. Chapman and Hall. New York.

[5] Quenouille , M. H., 1956. Notes on Bias in Estimation, Biometrika, 43: 353-360

[6] Yu, C. H., 2003. Resampling Methods: Concepts, Applications and Justification, Practical Assessment, Research-Evaluaiton, $8(19)$.

[7] Akşar, T., (2011). Parliamentary Minutes of Meeting Related To Sport Clubs' Financial Administration Issues and Solution Offers.03.2011, http://www.futbolekonomi.com,

(E.T. 30.04.2013).

[8] Dimitropoulos, P., (2010). The Financial Performance of the Greek Football Clubs, Sport Management International Journal, SMIJ, Vol. 6, Nr. 1, ss. 5-27.

[9] Ulusoy, O., 2014. Financial Performance Analysis of Super League Football Clubs. Journal of Yasar University. 9(34) 5716-5731.

[10] Efron, B., 1979. Bootstrap Methods: Another look at jakknife. The annals of Statistics, 7(1): 1-26.

[11] Efron, B., 1981. Censored Data and Bootstrap, Journal of the Acoustical Society of America, 76(374), 312-319

[12] Hamajima, N., Yuasa, H., Matsuo, K., 1999. Methods for Statistical Inferences, Biotherapy 13(6), 739, 1999.

[13] Wehrens, R., Putter, H., Buydens, L. M. C., 2000. The Bootstrap: A Tutorial, Chemometrics and Intelligent Laboratory System. 54, 35-52.

[14] Tukey J. W., 1958. Bias and Confidence in Not-Quite Large Samples. The Annals of Mathematical Statistics. 29(2), 614.

[15] Walsh, B., 2000. Resampling Methods: Randomization Tests, Jakknife and Bootstrap Estimators. Lecture Notes for EEB $596 z$.

[16] http://www.math.ntu.edu.tw/ hchen/teaching/LargeSample/ref erences/Rbootstrap.pdf. Visit date: September 3, 2014.

[17] http://www.math.montana.edu/ parker/PattersonStats/Bootstra p.pdf Visit date : September 3, 2014. 